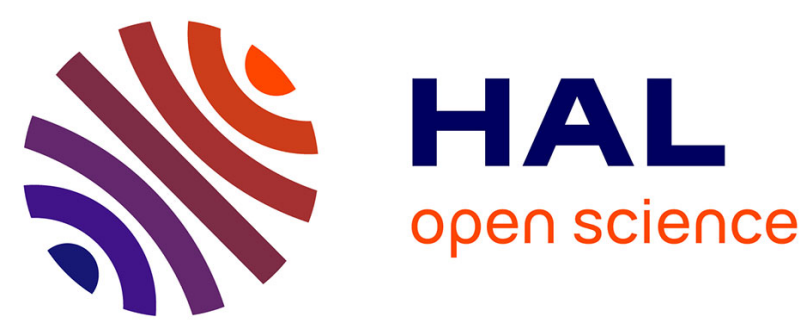

\title{
Muscle activation during cycling at different cadences: Effect of maximal strength capacity
}

\author{
François Bieuzen, Romuald Lepers, Fabrice Vercruyssen, Christophe
}

Hausswirth, Jeanick Brisswalter

\section{To cite this version:}

François Bieuzen, Romuald Lepers, Fabrice Vercruyssen, Christophe Hausswirth, Jeanick Brisswalter. Muscle activation during cycling at different cadences: Effect of maximal strength capacity. Journal of Electromyography and Kinesiology, 2007, 17 (6), pp.731-738. 10.1016/j.jelekin.2006.07.007 . hal01708179

\section{HAL Id: hal-01708179 https://hal-insep.archives-ouvertes.fr/hal-01708179}

Submitted on 13 Feb 2018

HAL is a multi-disciplinary open access archive for the deposit and dissemination of scientific research documents, whether they are published or not. The documents may come from teaching and research institutions in France or abroad, or from public or private research centers.
L'archive ouverte pluridisciplinaire HAL, est destinée au dépôt et à la diffusion de documents scientifiques de niveau recherche, publiés ou non, émanant des établissements d'enseignement et de recherche français ou étrangers, des laboratoires publics ou privés. 
Muscle activation during cycling at different cadences: Effect of maximal strength capacity

François Bieuzen', Romuald Lepers², Fabrice Vercruyssen', Christophe Hausswirth³ Jeanick Brisswalter ${ }^{1}$

' Laboratoire d'Ergonomie Sportive, EA 3162, Université du Sud Toulon-Var, France

2 INSERM ERM207, UFR STAPS, Université de Bourgogne, Dijon Cedex, France

${ }^{3}$ Laboratoire de Physiologie et Biomécanique, INSEP, France

() Pr. J.Brisswalter, Laboratoire d'Ergonomie Sportive, Université de Toulon-Var, Avenue de I'Université, BP 132, 83957 LA GARDE cedex, France.

Fax (0033) 494142278 
ABSTRACT

The purpose of this study was to examine the influence of maximal strength capacity on muscle activation, during cycling, at three selected cadences: a low cadence $(50 \mathrm{rpm})$, a high cadence $(110 \mathrm{rpm})$ and the freely chosen cadence (FCC). Two groups of trained cyclists were selected on the basis of the different maximal isokinetic voluntary contraction values (MVCi) of their lower extremity muscles as follow: Fmin (lower MVCi group) and Fmax (higher MVCi group). All subjects performed three 4-min cycling exercises at a power output corresponding to $80 \%$ of the ventilatory threshold under the three cadences. Neuromuscular activity of vastus lateralis (VL), rectus femoris (RF) and biceps femoris (BF) was studied quantitatively (integrated electromyography, IEMG) and qualitatively (timing of muscle bursts during crank cycle). Cadence effects were observed on the EMG activity of VL muscle and on the burst onset of the BF, VL and RF muscles. A greater normalized EMG activity of $\mathrm{VL}$ muscle was observed for the $\mathrm{F}_{\min }$ group than the $\mathrm{F}_{\max }$ group at all cadences (respectively Fmin vs. Fmax at 50 rpm: $17 \% \pm 5$ vs. $38 \% \pm 6$, FCC: $22 \% \pm 7$ vs. $44 \% \pm 5$ and $110 \mathrm{rpm}: 21 \% \pm 6$ vs. $45 \% \pm 6)$. At FCC and $110 \mathrm{rpm}$, the burst onset of BF and RF muscles of the $F_{\max }$ group started earlier in the crank cycle than the Fmin group These results indicate that in addition to the cadence, the maximal strength capacity influences the lower extremity muscular activity during cycling.

Key words: cyclists, freely chosen cadence, torque, EMG, force.

(Article paru dans: Journal of electromyography and kinesiology, dec 2007, vol. 17, n6, pp. 731-738 


\section{Introduction}

The underlying reasons leading to the choice of a particular pedaling cadence (freely chosen cadence, FCC) in cyclists have not yet been clearly established. Because the minimization of oxygen cost during cycling cannot be considered as the main factor determining FCC (e.g. Brisswalter et al, 2000), neuromuscular hypotheses have also been suggested to explain the choice of FCC, postulating that cycling at FCC could minimize electromyography (EMG) activity. For example, Takaishi et al (1996) reported that FCC could minimize neuromuscular fatigue of the vastus lateralis muscle. On the other hand, in a modelization study, Neptune et al. (1999) demonstrated that muscular activity was minimized at $90 \mathrm{rpm}$ in order to decrease muscle force applied on the crank. In contrast, Sarre et al (2003) showed that in trained subjects, the neuromuscular activity of the knee extensor muscles was not significantly influenced by the changes in cadence, irrespective of the level of power output. It appears therefore that the influence of cadence on the level of EMG depends on the muscle considered and its functional role.

Currently, muscle activity on a range of selective cadence is quantitatively analyzed using EMG values as the dependant variable (e.g. Baum et al, 2003; Billaut et al, 2005; Neptune et al, 1997). Furthermore, many studies have proposed that muscle activation (burst onset, offset and duration) could be used to examine how the central nervous system responds to anatomical and geometrical constraints, and could therefore identify an efficient motor pattern (e.g. Li, 2004; Prilutsky et al, 2000; Van Ingen Schenau, 1989a, 1989b). Previous studies showed that EMG burst onset values shift earlier in the crank cycle with increasing cadences in cycling (e.g. Baum et al, 2003; Neptune et al, 1997; Sarre et al, 2005). In order to explain these results, Neptune et al. (1997) proposed the "activation dynamics hypothesis" suggesting 
that these coordination changes may allow subjects to develop torques in the same relative area of the crank cycle.

Previous work has shown that FCC varied widely among subjects, with the same training status but different experience in cycling (e.g. Marsh et al, 1995, 1997). These authors hypothesized that the similar preferred cadences of trained cyclists and runners compared to less trained subjects were related to muscle characteristics, in particular to similarities in the force-velocity (F-V) properties of the lower extremity muscles developed during endurance training (i.e. high repetitions or relatively fast joint angular velocities).

For each cadence at a given power output, there is a corresponding mean torque value and therefore a specific force applied on the pedals. This mean torque or force corresponds to a percentage of the maximum strength capacity that differs between subjects. Indeed, during cycling at the same cadence and power output, the corresponding mean torque will correspond to a lower percentage of maximal force capacity for the stronger cyclists. Therefore, the maximal strength capacity of cyclists could influence the level of neuromuscular activity when riding at a given cadence and power output levels. To our knowledge, no previous study has examined the relation between muscle activation and strength capacity of cyclists. We hypothesized that muscle activation during cycling might be influenced by the level of strength.

In this context, the purpose of the present study was to examine the influence of maximal strength capacity of leg muscles on EMG activity and muscle coordination when cycling at three different cadences: a low cadence (50 rpm), a high cadence (110 rpm) and the freely chosen cadence (FCC) in two groups of trained cyclists characterized by different maximal strength capacities. 


\section{Methods}

\subsection{Participants}

Twenty-four trained and motivated male cyclists (age: $32 \pm 5$ years; mass: $72 \pm$ $8 \mathrm{~kg}$; height: $177 \pm 6 \mathrm{~cm}$ ) who currently took part in cycling competition at a national level participated in this study. Subjects were fully informed about the protocol, and informed consent was obtained prior to all testing. This study was approved by a local research ethics committee. Each subject completed three laboratory-based testing sessions separated by at least a 48 hour rest period.

\subsection{Test procedure}

The aim of the initial session was to evaluate the maximal isokinetic voluntary contraction level (MVCi) of the lower extremity limb during a squat movement conducted on a specific ergometer (Ariel Computerized Exercise System (ACES) « multifunction exercise », Ariel Dynamics Inc., Trabucco Canyon, USA). The range of motion was standardized so that the movement started from a start position with a trunk/thigh and knee angles of $90^{\circ}$ and finished upright with an extended lower limb $\left(\right.$ knee angle $\left.=180^{\circ}\right)$. Each subject performed three sets of two repetitions of maximal isokinetic squat at two different velocities $\left(16\right.$ and $\left.8 \mathrm{~cm} \cdot \mathrm{s}^{-1}\right)$. A short rest time was imposed between each repetition (30 sec) and each set (5 min). The subjects were instructed to push the bar "as fast as possible", and they were encouraged during the whole movement. The maximal peak force values were obtained either for isokinetic velocities of 16 or $8 \mathrm{~cm} \cdot \mathrm{s}^{-1}$. Subsequently, the subjects were divided in two groups $(2 \times 12)$ based on their MVCi values (Table 1) as follow: Fmin (lower MVCi group) and $F_{\max }$ (higher MVCi group). For all subjects, the right leg was their 
dominant leg, as determined by kicking a ball according to the method described by Daly and Cavanagh (1976).

During the second session, subjects had to perform an incremental cycling test at a self-selected cadence on an electromagnetically braked ergocycle (Excalibur sport, Lode, Gröningen, The Nederland). The handlebars and racing seat are fully adjustable both vertically and horizontally to reproduce conditions known from the subjects' own bicycles. Moreover, this ergometer is equipped with individual racing pedals and toes clips allowing subject to wear their own cycling shoes. The ergometer allows subjects to maintain the power output constant independent of the selected cadence, by automatically adjusting torque to angular velocity. The test began with a warm-up at $100 \mathrm{~W}$ lasting 6-min, after which the power output was increased by $30 \mathrm{~W}$ each minute until the subjects were exhausted. The criteria used for the determination of maximal oxygen uptake ( $\left.\dot{\mathrm{V}} \mathrm{O}_{2 \mathrm{max}}\right)$ were a plateau in $\dot{\mathrm{V}} \mathrm{O}_{2}$ despite an increase in workrate and a respiratory exchange ratio (RER) above 1.1 or a heart rate (HR) over $90 \%$ of the predicted maximal HR (e.g. Howley et al, 1995). The four highest consecutive $\dot{\mathrm{VO}}_{2}$ values were summed in the last stage to determine $\dot{\mathrm{V}} \mathrm{O}_{2 \text { max. }}$ The ventilatory threshold (VT) was determined by using the criteria of an increase in $\dot{\mathrm{V}}_{\mathrm{E}} / \dot{\mathrm{VO}}_{2}$ with non-concomitant increase in $\dot{\mathrm{V}}_{\mathrm{E}} / \dot{\mathrm{VCO}}_{2}$ (e.g. Wassermann et al, 1973).

Before starting the third session, subjects were placed in a seated position and were securely strapped into the test chair to perform a right isometric knee extension and flexion using an isometric ergometer (Schnell Trainingsgesäte $\mathrm{GmbH}$, Peutenhausen, Deutschland). The studied limb was the right leg. Subjects sat with a knee angle of $90^{\circ}\left(0^{\circ}\right.$ being full leg extension), with the ankle attached to the ergometer arm. The knee axis was aligned with the dynamometer axis. EMG was recorded on the vastus lateralis (VL) and the rectus femoris (RF) muscles during the 
maximal voluntary contraction of the knee extensors (MVC), and on the biceps femoris (BF) during the MVC of the knee flexors. Subjects performed two maximal isometric contraction of short duration $(2-3 \mathrm{sec})$ of the knee flexor and extensor muscles. A 60-s period of rest was imposed between each contraction. The maximal force values of knee extension and flexion movement were measured using a strength sensor and the best of the two trials was selected as the maximal isometric voluntary contraction (MVC, in Newton). Maximal integrated EMG values were calculated for VL, RF and BF muscles during MVC (period of $500 \mathrm{~ms}$ ) for the knee flexion exercise, and were used to normalize the neuromuscular activity recorded during cycling.

Subsequently, subjects performed a constant cycling exercise (12 min) conducted at three assigned cadences (4 min at 50, $110 \mathrm{rpm}$ and FCC) and at a power output corresponding to $80 \%$ of the workrate reached at VT. The cadences were presented in a random order and no rest was allowed between the three trials. A computer with a cadence monitor provided feedback to the subjects so that the cadence of 50 or $110 \mathrm{rpm}$ could be maintained within $\pm 1 \mathrm{rpm}$ of the target cadence for the whole duration of the trial. During the FCC trial, the cadence monitor was covered and the subject had to choose a cadence considered as the most comfortable. The cadence was continuously monitored during the test. During the overall cycling bout, pulmonary gas exchanges were measured using a portable telemetric gas exchange system (Cosmed $\mathrm{K} 4 \mathrm{RQ}{ }^{\circledR}$, Rome, Italy) previously validated by Hausswirth et al. (1997) and were recorded during the fourth minute of each trial.

\subsection{Muscle activity}

The muscles activities of the VL, RF and BF muscles of the right leg, selected for their high contribution to the propulsive cycling task (e.g. Ryan et al, 1992), were 
monitored with surface EMG. The subjects were prepared for the placement of EMG electrodes by shaving the skin of each electrode site, cleaning it carefully with alcohol wipe and lightly abrading it to maintain a low inter-electrode resistance of $<1000 \Omega$. Pairs of Ag/AgCl pre-gelled surface electrodes (Blve Sensor Q-00-S, Medicotest, Denmark) of $40 \mathrm{~mm}$ diameter with a center to center distance of $25 \mathrm{~mm}$ were applied along the fibers over the bellies of the three muscles for EMG data acquisition. The electrodes were secured with surgical tape and cloth wrap to minimize disruption during the movement. A ground electrode was placed on a bony site over the right anterior superior spine of the iliac crest.

\subsection{Data collection and processing}

EMG signals were pre-amplified close to detection site (Common Mode Rejection Ratio, $C M R R=100 \mathrm{~dB} ; \mathrm{Z}$ input = $10 \mathrm{G} \Omega ;$ gain = 600, bandwidth frequency $=$ from $6 \mathrm{~Hz}$ to $1600 \mathrm{~Hz}$ ). The signals were then filtered at $500 \mathrm{~Hz}$ with a third order, zero lag Butterworth antialiasing filter and digitized at $1000 \mathrm{~Hz}$ by using an acquisition board (DT 9800-series, Data Translation, Marlboro, USA). Subsequent analyses were performed with custom-written add-on software (Origin 6.1 ${ }^{\circledR}$, OriginLab, Northampton, USA, EMG Toolbar add-on).

EMG data were collected from each muscle during 40 consecutive crank cycles within the last minutes of the trials at each cadence and were normalized (normalized EMG) according to muscle maximal EMG obtained during MVC test for each individual muscle.

Three discrete event parameters were then calculated and automatically detected with a specific software (Origin 6.1®, OriginLab, Northampton, USA): EMG burst onset, offset and EMG burst duration. Before calculating these parameters, raw data were unbiased, full-wave-rectified, and then smoothed with a FFT low pass filter 
at $10 \mathrm{~Hz}$, to create a linear envelope. The criteria for the onset and the offset values were based on a minimum threshold of 3 standard deviations from the resting baseline and a minimum burst duration of $50 \mathrm{~ms}$ according to the study of Neptune et al. (1997). Upon reaching the determined threshold, the muscle was considered active, and the muscle "burst" duration was defined as the duration between the onset and offset values. The end of the second muscle burst was considered as muscle deactivation when the subject exhibited a double-burst pattern according to the study of Sarre and Lepers (2005). Burst onset and offset timing and burst duration were expressed as a percentage of the total duration between two consecutive peak torques.

\subsection{Torque measurement}

The mechanical torque during pedaling was measured with the cycling ergometer. The strain-gauge force transducer equipped inside the crank produced an analog signal that indicated the magnitude of the force perpendicular to the pedal (torque). Electric signals from a DC amplifier were simultaneously recorded with EMG signal on the data recorder. The crank angle value was provided by the cycle ergometer. When the right crank arm was at the top-dead centre, the crank angle was $0^{\circ}$. The mean peak torque for each cadence was determined by the average of the peak torque values recorded during the same period as EMG recording.

\subsection{Statistical analysis}

All data were expressed as mean \pm standard deviation (SD). A two-way analysis of variance (group $x$ cadence) for repeated measures was performed to 
analyze the effect of groups and cycling cadences by using physiological, biomechanical and EMG values as dependent variables. Tukey post hoc test was used to determine any differences among the cycling cadences and groups during exercise. A non-parametric test (Wilcoxon) was performed to select two significantly different groups from their MVCi values. Furthermore, trend analyses were performed wherever applicable to identify significant trends for cadence. The 0.05 level of significance was used for all statistical procedures.

\section{Results}

Table 1 shows the mean values of $\mathrm{MVCi} \dot{\mathrm{V}} \mathrm{O}_{\text {2max, }} \mathrm{HR}$ max, maximal aerobic power (MAP) and power output at the ventilatory threshold (VTp) for the two groups. The MVCi values were significantly different between the two experimental groups $\left(1415 \pm 46 \mathrm{~N}\right.$ and $1993 \pm 177 \mathrm{~N}$, respectively for the $F_{\min }$ and $F_{\max }$ groups, $\left.P<0.05\right)$. No significant effect of group was found on $\dot{\mathrm{V}}_{2 \text { max, }}$ MAP, VTP and FCC values.

Right peak torque and the corresponding crank angle for the two groups are shown in Table 2. No significant differences between groups were found at each cadence. For each experimental group, a significant cadence effect $(p<0.05)$ was found on the right peak torque and its crank angle. Right peak torque was significantly lower at $110 \mathrm{rpm}$ and FCC than at $50 \mathrm{rpm}$ and it occurred significantly later in the cycle at $110 \mathrm{rpm}$ and FCC than at $50 \mathrm{rpm}$.

\section{1. Normalized EMG during cycling}

Normalized EMG activity of the BF and RF muscles were not significantly $(p<0.05)$ affected by cadence (fig. 1). In contrast, normalized EMG of VL muscle was significantly lower at $50 \mathrm{rpm}$ compared to $\mathrm{FCC}$ and $110 \mathrm{rpm}$. A significant $(p<0.05)$ 
group effect was found on EMG activity of VL muscle only: it was lower for the $F_{\max }$ group when compared to the $F_{\min }$ group at 50, FCC and $110 \mathrm{rpm}$. No group effect was observed on RF and BF muscles.

\subsection{Burst onset and offset}

The patterns of all muscles activity across cadence and group conditions are displayed on Fig. 2. As pedaling cadence increased, BF, VL and RF showed significant $(p<0.05)$ changes in crank cycle of muscle burst onset and offset. Among these muscles and groups, all but the burst onset of RF of Fmin group exhibited a significant linear trend $(p<0.05)$ with the onset and offset timing shifting to an earlier time with increased cadence. Significant differences $(p<0.05)$ in burst onset and offset due to MVCi conditions were observed in BF and RF at $110 \mathrm{rpm}$ and FCC. Specifically, burst onset and offset of Fmax group for BF and RF muscles respectively started and ended significantly earlier $(p<0.05)$ in the crank cycle than in the Fmin group at 110 rpm and FCC. No significant difference $(p<0.05)$ in the offset timing between groups was observed on VL. No significant difference $(p<0.05)$ in the onset and offset timing between groups was observed at $50 \mathrm{rpm}$ on BF, VL and RF.

\subsection{Burst duration}

The burst duration of VL and RF muscles displayed no significant differences $(p<0.05)$ according to cadence. On BF muscle, burst duration was significantly longer at 50 rpm compared to FCC and 110 rpm for each group.

Whatever the cadence, no significant $(p<0.05)$ group effect was observed on burst duration of BF, VL and RF muscles. 


\section{Discussion}

The purpose of the present study was to investigate the interaction between maximal strength capacity and the muscle activation at three pedaling rates: 50 rpm, FCC and $110 \mathrm{rpm}$. The results showed that the neuromuscular activity of the VL muscle was influenced by the maximal strength level, with a higher EMG activity for the Fmin group than the Fmax group. Concerning the muscle activation pattern, it appeared that at FCC and 110 rpm, the EMG burst onset of BF and RF muscles of the Fmax group started earlier in the crank cycle than the Fmin group.

The first interesting result of this study was the differences observed between 50, FCC and $110 \mathrm{rpm}$. At FCC and $110 \mathrm{rpm}$ compared to $50 \mathrm{rpm}$, our results showed greater normalized EMG values of VL muscle associated with a decrease in the right peak torque that occurred later in the crank cycle. Furthermore, whatever the muscle investigated, we have observed a systematic shift to an earlier time of bursts onset and offset with increasing cadence. Finally, burst duration of the BF muscle was shorter at FCC and $110 \mathrm{rpm}$ compared to $50 \mathrm{rpm}$. Many reasons detailed below could explain these observations.

Neuromuscular activities of the BF, VL and RF muscles were differently affected by cadence selection. In the present study, the EMG activity of the VL muscle increased with cadence whereas the EMG of the BF and RF muscles remained unchanged. Our results corroborate these of Marsh and Martin (1995) who observed similar results on the $\mathrm{VL}$ muscle with an increase in IEMG values with increasing cadence. In contrast to previous studies (e.g. Marsh et al, 1995; Neptune et al, 1997; Takaishi et al, 1998), a minimization of the EMG activity at FCC was not found in the present study. Furthermore, the EMG activity of BF and RF muscles did not change with cadence in accordance with previous results for low power output (<250W) (e.g. Neptune et al, 1997; Maclntosh et al, 2000; Sarre et al, 2003). These 
results could be related to those observed by Citterio and Agostini (1984) who suggested that there was a derecruitment of slow motors units with a recruitment of a smaller number of fast ones when the speed of movement is increased at constant power output. Thus, a possible shift in recruitment within each muscle based on fiber characteristics could have occurred here.

Comparing quantitative EMG activity against maximal strength level showed an interesting finding. For the VL muscle, the EMG activity of the Fmin group was twice higher than the Fmax group at each cadence. This original result could be related to the fact that for the weaker cyclists the corresponding mean torque will correspond to a greater percentage of maximal force capacity and therefore a greater percentage of maximal neuromuscular activity. In contrast the EMG activity of BF and RF muscles was not influenced by the level of strength. This observation showed that the neuromuscular activity of the RF and BF biarticular muscles during cycling was independent of the maximal strength of the subjects. This difference might be explained by the anatomical specificity of each muscle. Indeed, the monoarticular vastii muscles produce the torque during the extension phase whereas the biarticular muscles propel the pedal crank through the top and bottom dead centre position (e.g. van Ingen Schenau et al, 1995).

Concerning muscle coordination, the results showed a shift of the burst onset that occurred earlier in the crank cycle with increasing cadence for the BF, VL and RF muscles, whatever the experimental group. This finding is on accordance with previous results (e.g. Neptune et al, 1997; Baum et al, 2003; Sarre et al, 2005) and could be explained by a phase advance to account for the activation dynamics rather than indicative of a change mechanical muscle function (e.g. Neptune et al, 1997). The EMG burst onset at FCC followed the general linear trends of the effect of cadence manipulation on muscular coordination and no specific pattern appeared at FCC. Concerning the relative burst duration, a significant decrease with increasing 
cadence was found for the antagonist BF muscle (e.g. Baum et al, 2003), while burst duration of the RF and VL muscles remained stable among cadences. It would seem that the increase in the speed of movement corrupts the muscular coordination by making it less efficient. So, on high cadences, the activation of BF would be weaker due to a less efficient muscular coordination. An original finding of the present study was the influence of maximal strength capacity on EMG activation pattern. Indeed, the burst onset of the BF and RF muscles in the Fmax group began significantly earlier in the crank cycle than for the Fmin group at $110 \mathrm{rpm}$ and FCC. It should be noted that these different patterns occurred between the two groups while no change in the mean peak torque (Fmin: $49 \pm 5$ N.m vs. Fmax: $52 \pm 11$ N.m) and in the corresponding crank angle (Fmin: $93 \pm 5^{\circ}$ vs. Fmax: $93 \pm 13^{\circ}$ ) was observed. Therefore, differences in EMG burst timing between groups could be mainly related to maximal strength capacity. In contrast to BF and RF muscles, the activation pattern of $\mathrm{VL}$ muscle was not influenced by the level of strength. One explanation for this lack of effect on VL muscle could be related to the anatomical specificity of this muscle. Previous studies (e.g. Baum et al, 2003; Raasch et al, 1999) have suggested that the role of a muscle during cycling movement depends on its anatomical specificity. Raasch and co-workers (1997) showed that one pair of muscle groups (the vastii muscles) produce the energy needed to propel the crank through limb extension and flexion, with some energy to accelerate the limb segments first. In contrast, the role of the RF and BF muscles is more complex. These muscles facilitate the transfer of energy to the crank produced by the other muscles and also produce energy to propel the crank directly, near the end of the extension and during the limb transition from extension to flexion (BF) and near the end of flexion and during the flexion-toextension transition (RF). Therefore, it appeared that at cadences higher than FCC stronger cyclists had an earlier burst onset in the crank cycle for bi-articular RF and BF muscles compared to weaker cyclists. Further investigations on greater number of 
muscles of the lower limb and at different power output are necessary to identify the factors that could explain this finding.

In conclusion, the present results suggested that maximal strength level could influence the muscle activation during cycling. The effects of the maximal strength capacity depended on the cadence and the role of the considered muscle. Indeed, the stronger the cyclist, the lower the EMG activity of the mono-articular VL muscle whereas it was unchanged for the BF and RF bi-articular muscles. On the contrary, when considering neuromuscular patterns, only bi-articular muscles were affected by the maximal strength level with the EMG burst onset starting earlier in the crank cycle for the stronger cyclists at high cadence (>FCC). Further studies are required to investigate the interaction between the maximal strength capacity, cadence and neuromuscular activities for pedaling exercise performed at different power outputs or for longer duration. 
Table 1 - Characteristics of the two experimental groups (mean \pm SD). $\dot{V} O_{2 m a x}$ : maximal oxygen uptake, MAP: maximal aerobic power output, $\mathrm{VT}_{\mathrm{p}}$ : Power output at the ventilatory threshold, FCC: Freely chosen cadence and MVCi: Maximal isokinetic voluntary contraction. $(*$, significantly different between the two groups $(P<0.05))$.

\begin{tabular}{|c|c|c|c|}
\hline & $F_{\min }(n=12)$ & $F_{\max }(n=12)$ & $\begin{array}{l}\text { Difference between } \\
\text { groups }\end{array}$ \\
\hline Mass (kg) & $67 \pm 8$ & $69 \pm 6$ & NS \\
\hline Height $(\mathrm{cm})$ & $174 \pm 7$ & $177 \pm 5$ & NS \\
\hline$\dot{V} \mathrm{O}_{2 \max }\left(\mathrm{mL} \cdot \mathrm{min}^{-1} \cdot \mathrm{kg}^{-1}\right)$ & $67 \pm 10$ & $69 \pm 7$ & NS \\
\hline MAP (W) & $391 \pm 29$ & $421 \pm 31$ & NS \\
\hline $\mathrm{VT}_{\mathrm{p}}(\mathrm{W})$ & $216 \pm 19$ & $225 \pm 26$ & NS \\
\hline FCC (rpm) & $87 \pm 6$ & $93 \pm 12$ & NS \\
\hline MVCi (N) & $1415 \pm 46$ & $1993 \pm 177$ & $*$ \\
\hline
\end{tabular}


Table 2 - Changes in mean right peak torque and corresponding crank angle for the two groups $\left(F_{\min }\right.$ and $\left.F_{\max }\right)$ at three cadences $(50,110 \mathrm{rpm}$ and $\mathrm{FCC})$. FCC: freely chosen cadence. $\left(0^{\circ}=\right.$ right pedal at top-dead centre $)$.

\section{Cadence}

(rpm)

50

FCC

110

Right peak torque

Fmin

$63 \pm 5$

$49^{*} \pm 5$

$43^{*} \pm 6$

(N.m)

Fmax

$69 \pm 14$

$52^{*} \pm 11$

$46^{*} \pm 7$

Crank angle of right

Fmin

$80 \pm 7$

$93^{*} \pm 5$

$93^{*} \pm 7$

peak torque

$\left({ }^{\circ}\right)$

Fmax $\quad 74 \pm 4 \quad 93^{*} \pm 13 . \quad 99^{*} \pm 9$

Group effect: No significant difference between the groups for all values.

Cadence effect: *, represents significant difference between 50 rpm $(p<0.05)$ 

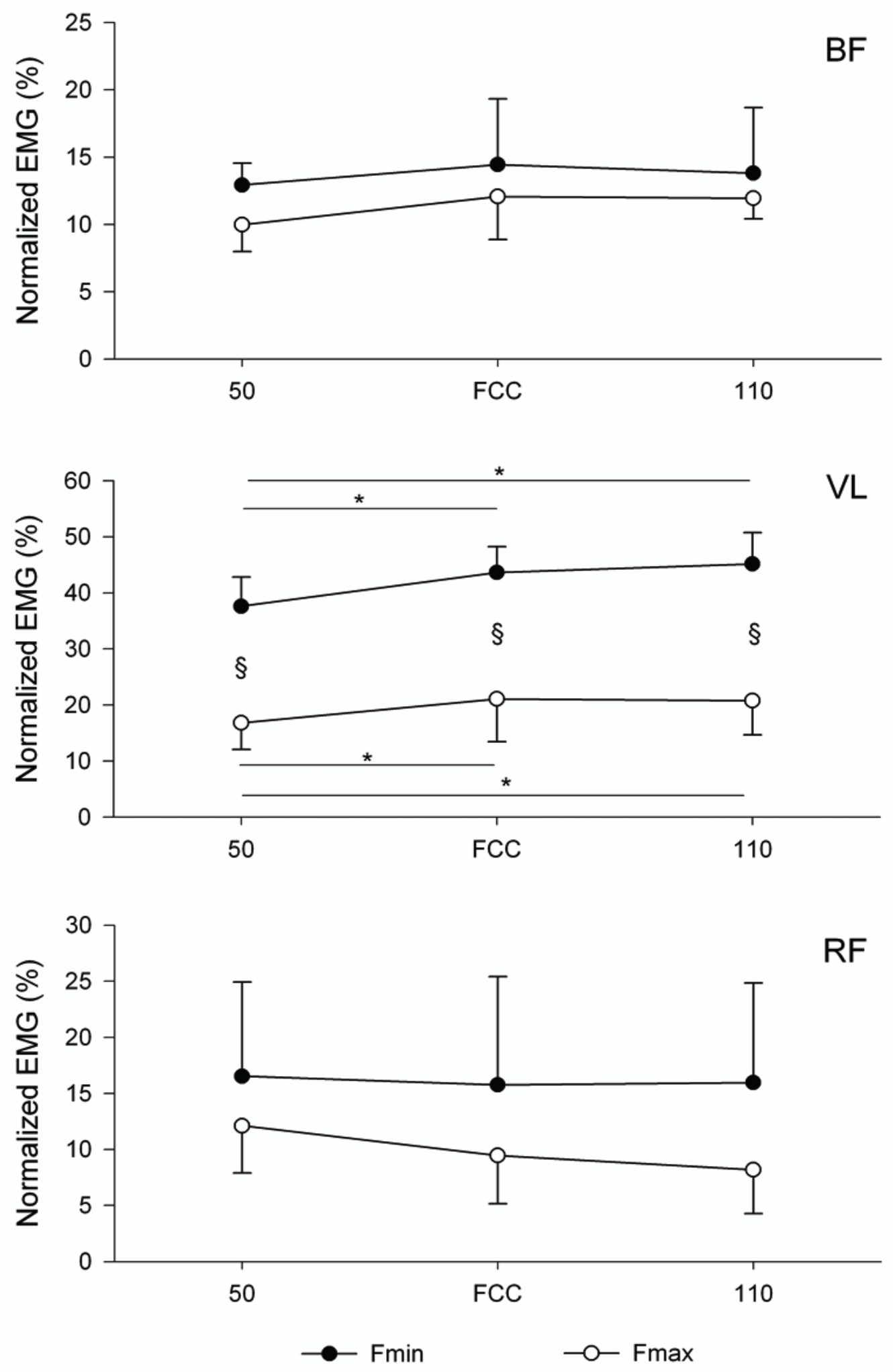

Fig. 1 - Influence of cadence on the level of neuromuscular activity of vastus lateralis $(V L)$, rectus femoris (RF) and biceps femoris $(B F)$ for the two groups ( $F_{\min }$ and $F_{\text {max }}$ ).

$*$, significant difference between cadence $(p<0.05)$

$\S$, significant difference between groups $(p<0.05)$ 


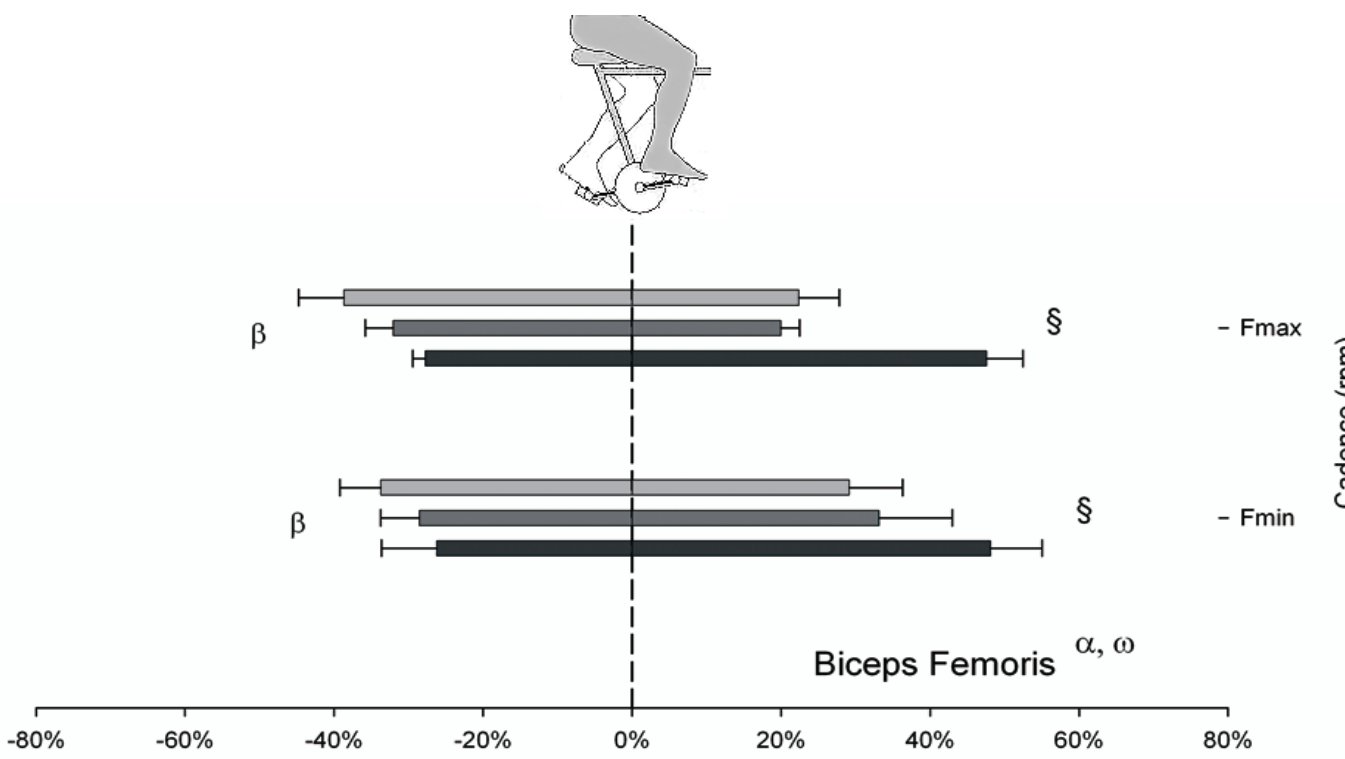

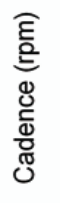

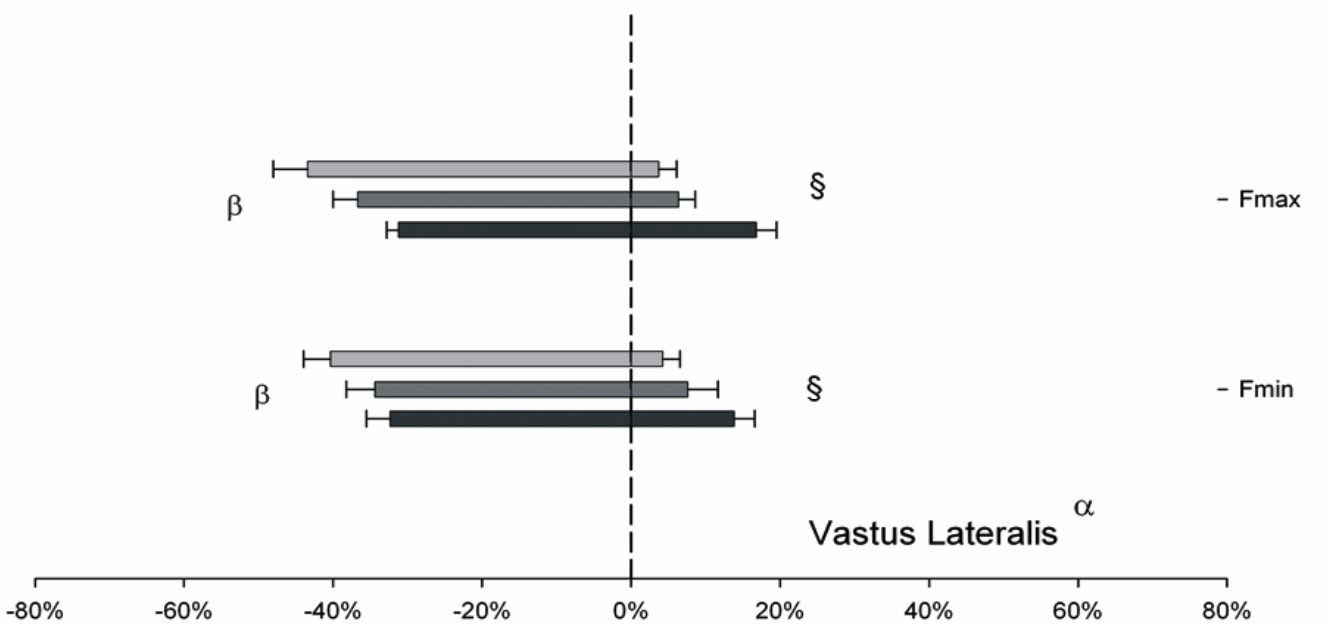

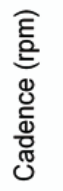

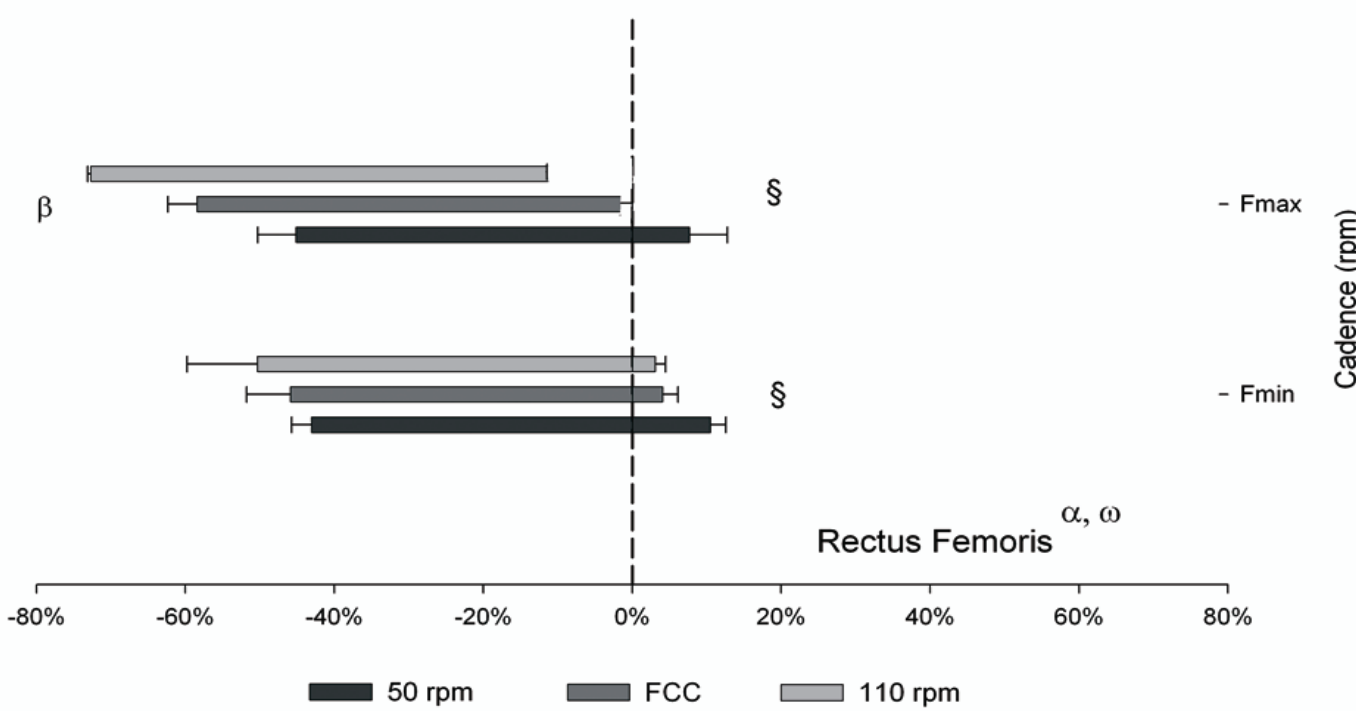

Fig. 2 Mean onset, offset, and duration of EMG linear envelopes of biceps femoris $(B F)$, vastus lateralis (VL) and rectus femoris (RF) across cadence and group conditions. Values were expressed as a percentage of the total duration between two consecutive peak torques. The left and right edges of each rectangle represent mean onset and offset values, respectively. Errors bars represent one standard deviation of the mean onset and offset. $B$ and $\S$ indicate a statistically significant difference $(p<0.05)$ between cadences $(50, \mathrm{FCC}$ and $110 \mathrm{rpm}$ ) for onset and offset 
respectively. a, $\omega$ indicate a statistically significant difference $(p<0.05)$ at $110 \mathrm{rpm}$ and FCC between group (Fmin and Fmax) for onset and offset respectively. 

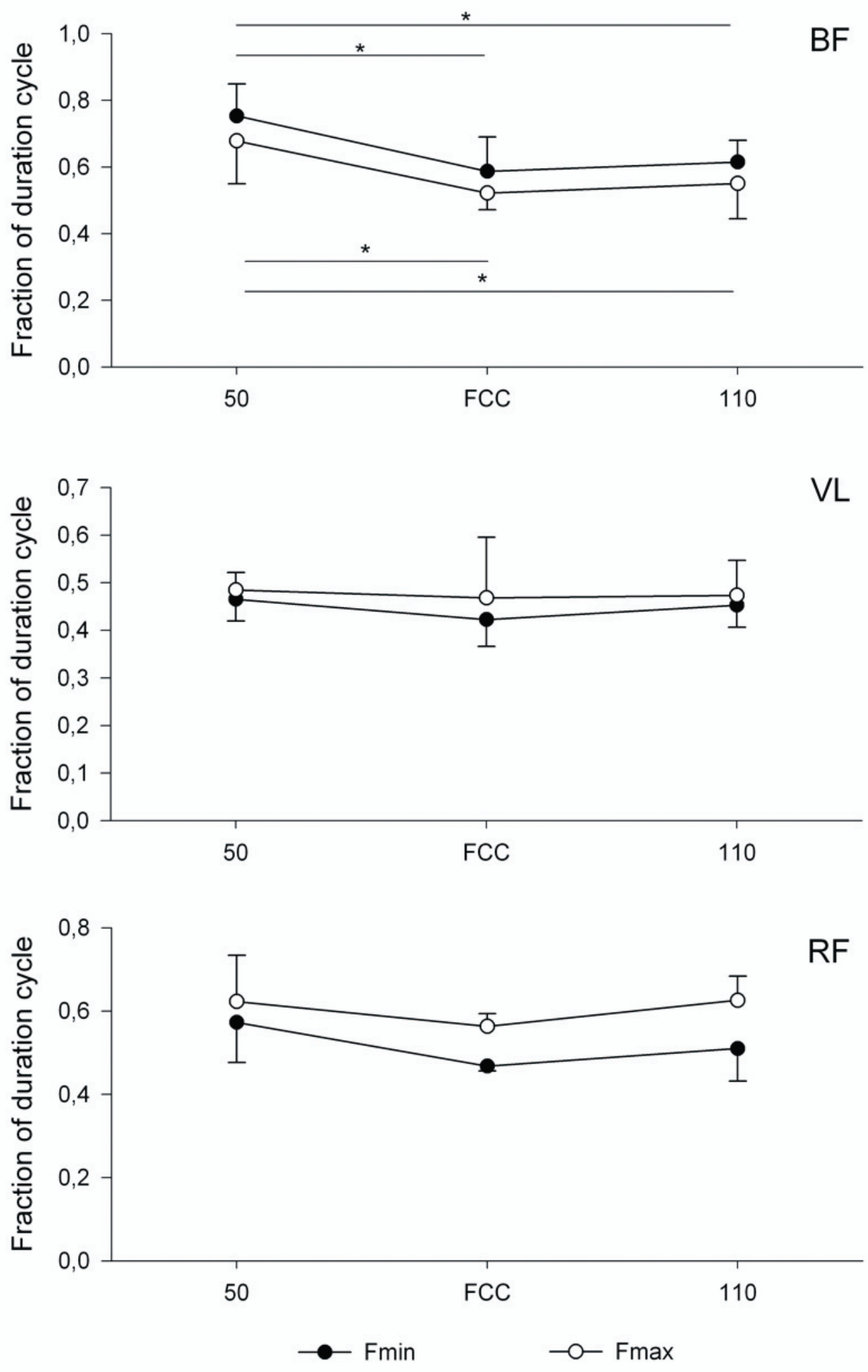

Fig. 3 - Influence of cadence on the EMG burst duration of vastus lateralis (VL), rectus femoris (RF) and biceps femoris (BF) for the two groups ( $F_{\min }$ and $\left.F_{\max }\right)$.

$*$, significant difference between cadence $(p<0.05)$

No significant difference between groups $(p<0.05)$ 


\section{References}

Baum BS, Li L. Lower extremity muscle activities during cycling are influenced by load and frequency. J Electromyogr Kinesiol 2003;13(2):181-90.

Billaut F, Basset FA, Falgairette G. Muscle coordination changes during intermittent cycling sprints. Neurosci Let† 2005;380(3):265-9.

Brisswalter J, Hausswirth C, Smith D, Vercruyssen F, Vallier JM. Energetically optimal cadence vs. freely-chosen cadence during cycling: effect of exercise duration. Int J Sports Med 2000;21 (1):60-4.

Citterio G, Agostoni E. Selective activation of quadriceps muscle fibers according to bicycling rate. J Appl Physiol 1984;57(2):371-9.

Daly DJ, Cavanagh PR. Asymmetry in bicycle ergometer pedaling. Med. Sci. Sports Exerc. 1976;8(3):204-8.

Hausswirth C, Bigard A, Le Chevalier JM. The Cosmed K4 telemetry system as an accurate device for oxygen uptake measurements during exercise. Int J Sports Med 1997;18(16):449-53.

Howley ET, Bassett DR Jr, Welch HG. Criteria for maximal oxygen uptake: review and commentary. Med Sci Sports Exerc 1995;27(9):1292-301.

Li L. Neuromuscular control and coordination during cycling. Res Q Exerc Sport $2004 ; 75(1): 16-22$.

Macintosh BR, Neptune RR, Horton JF. Cadence, power, and muscle activation in cycle ergometry. Med Sci Sports Exerc 2000;32(7):1281-7.

Marsh AP, Martin PE. The relationship between cadence and lower extremity EMG in cyclists and noncyclists. Med Sci Sports Exerc 1995;27(2):217-25.

Marsh AP, Martin PE. Effect of cycling experience, aerobic power, and power output on preferred and most economical cycling cadences. Med Sci Sports Exerc 1997;29(9):1225-32. 
Neptune RR, Kautz SA, Hull ML. The effect of pedaling rate on coordination in cycling. J Biomech 1997;30(10):1051-8.

Neptune RR, Hull ML. A theoretical analysis of preferred pedaling rate selection in endurance cycling. J Biomech 1999;32(4):409-15.

Prilutsky BI, Gregory RJ. Analysis of muscle coordination strategies in cycling. IEEE Trans Rehabil Eng 2000;8(3):362-70.

Raasch CC, Zajac FE, Ma B, Levine WS. Muscle coordination of maximum-speed pedaling. J Biomech 1997;30(6):595-602.

Raasch CC, Zajac FE. Locomotor strategy for pedalling: muscle groups and biomechanical functions. J Neurophysiol 1999;82(2):515-25.

Ryan MM, Gregor RJ. EMG profiles of lower extremity muscles during cycling at constant workload and cadence. J Electromyogr Kinesiol 1992;2:69-80.

Sarre G, Lepers R, Maffiuletti N, Millet G, Martin A. Influence of cycling cadence on neuromuscular activity of the knee extensors. Eur J Appl Physiol 2003;88(4$5): 476-9$.

Sarre G, Lepers R. Neuromuscular function during prolonged pedaling exercise at different cadences. Acta Physiol Scand 2005;185(4):321-8.

Takaishi T, Yasuda Y, Ono T, Moritani T. Optimal pedalling rate estimated from neuromuscular fatigue for cyclists. Med Sci Sports Exerc 1996;28(12):1492-7.

Takaishi T, Yamamoto T, Ono T, Ito T, Moritani T. Neuromuscular, metabolic, and kinetic adaptations for skilled pedaling performance in cyclists. Med Sci Sports Exerc 1998;30(3):442-9

Van Ingen Schenau GJ. From rotation to translation: constraints on multi-joint movements and the unique action of bi-articular muscles. Hum Mov Sci $1989 a ; 8: 301-37$

Van Ingen Schenau GJ. From rotation to translation: implications for theories of motor control. Hum Mov Sci 1989b;8:423-42. 
Van Ingen Schenau GJ, Dorssers WM, Welter TG, Beelen A, de Groot G, Jacobs R. The control of mono-articular muscles in multijoint leg extensions in man. J Physiol 1995:484(P† 1):247-54.

Wasserman K, Whipp BJ, Koyl SN, Beaver WL. Anaerobic threshold and respiratory gas exchange during exercise. J Appl Physiol 1973;35(2):236-43.

François Bieuzen is Ph.D student in the University of Toulon, France. His research interests focus on the relationship between muscular factors and energy cost of locomotion.

Romuald Lepers is Assistant Professor in the National Institute of Health and Medical Research at the University of Dijon in Burgundy, France. His research interests focus on the mechanisms of human fatigue during

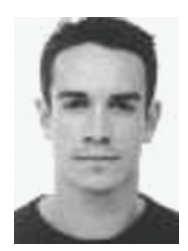
prolonged exercises such as cycling and running, and the neuromuscular adaptations to sustained submaximal contractions.

Fabrice Vercruyssen is Assistant Professor at the University of Toulon, France. His research interests focus on the relationship between neuromuscular fatigue and energy cost of locomotion.

Christophe Hausswirth is researcher at the national Institute for Sports and physical education in Paris, France. His research interests focus on the mechanisms underlying performance especially in elite athletes and

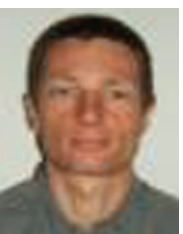
triathletes.

Jeanick Brisswalter is Professor and Head of the Department of Ergonomics at the University of Toulon, France. His research interests focus on the mechanisms underlying the energy cost of locomotion during

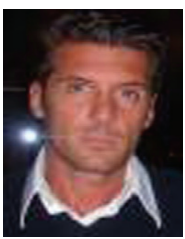
prolonged exercises. 
